\title{
Lactoferrin-modified rotigotine nanoparticles for enhanced nose-to-brain delivery: LESA-MS/MS- based drug biodistribution, pharmacodynamics, and neuroprotective effects
}

This article was published in the following Dove Press journal:

International Journal of Nanomedicine

\section{Xiuju Yan ${ }^{1, *}$ \\ Lixiao $\mathrm{Xu}^{1}{ }^{1, *}$ \\ Chenchen $\mathrm{Bi}^{1}$ \\ Dongyu Duan' \\ Liuxiang $\mathrm{Chu}^{\prime}$ \\ Xin Yu' \\ Zimei Wu' \\ Aiping Wang ${ }^{1,2}$ \\ Kaoxiang Sun ${ }^{1,2}$}

'School of Pharmacy, Collaborative Innovation Center of Advanced

Drug Delivery System and Biotech

Drugs in Universities of Shandong,

Key Laboratory of Molecular

Pharmacology and Drug Evaluation

(Yantai University), Ministry of

Education, Yantai University, Yantai,

Shandong Province, ${ }^{2}$ State Key

Laboratory of Long-Acting and

Targeting Drug Delivery System,

Shandong Luye Pharmaceutical

Co., Ltd, Yantai, Shandong Province,

People's Republic of China

*These authors contributed equally to this work

\begin{abstract}
Introduction: Efficient delivery of rotigotine into the brain is crucial for obtaining maximum therapeutic efficacy for Parkinson's disease (PD). Therefore, in the present study, we prepared lactoferrin-modified rotigotine nanoparticles (Lf-R-NPs) and studied their biodistribution, pharmacodynamics, and neuroprotective effects following nose-to-brain delivery in the rat 6-hydroxydopamine model of PD.
\end{abstract}

Materials and methods: The biodistribution of rotigotine nanoparticles (R-NPs) and Lf-R-NPs after intranasal administration was assessed by liquid extraction surface analysis coupled with tandem mass spectrometry. Contralateral rotations were quantified to evaluate pharmacodynamics. Tyrosine hydroxylase and dopamine transporter immunohistochemistry were performed to compare the neuroprotective effects of levodopa, R-NPs, and Lf-R-NPs.

Results: Liquid extraction surface analysis coupled with tandem mass spectrometry analysis, used to examine rotigotine biodistribution, showed that Lf-R-NPs more efficiently supplied rotigotine to the brain (with a greater sustained amount of the drug delivered to this organ, and with more effective targeting to the striatum) than R-NPs. The pharmacodynamic study revealed a significant difference $(P<0.05)$ in contralateral rotations between rats treated with Lf-R-NPs and those treated with R-NPs. Furthermore, Lf-R-NPs significantly alleviated nigrostriatal dopaminergic neurodegeneration in the rat model of 6-hydroxydopamine-induced PD.

Conclusion: Our findings show that Lf-R-NPs deliver rotigotine more efficiently to the brain, thereby enhancing efficacy. Therefore, Lf-R-NPs might have therapeutic potential for the treatment of PD.

Keywords: lactoferrin-modified rotigotine nanoparticles, nose to brain, drug biodistribution, pharmacodynamics, neuroprotective effects, Parkinson's disease

\section{Introduction}

Parkinson's disease (PD) is a neurodegenerative disorder characterized by the progressive and selective deterioration of nigrostriatal dopaminergic neurons, resulting in a lack of the neurotransmitter dopamine (DA) ${ }^{1-3}$ The disease affects more than $3 \%$ of people over the age of $65 .^{2}$ The current therapeutic strategy is to increase dopamine levels in the brain. Levodopa (L-DOPA) has remained the most important drug for the treatment of PD symptoms for over 30 years. However, long-term treatment with L-DOPA is associated with side effects, such as wearing-off and on-off phenomena, delayed onset of action, and the development of a disabling dyskinesia termed levodopa-induced dyskinesia. ${ }^{4}$ Thus, novel approaches, including new drugs
Correspondence: Kaoxiang Sun; Aiping Wang

School of Pharmacy, Yantai University, No 30 Qingquan Road, Yantai 264005, Shandong Province, People's Republic of China

Tel +86535394 6400; +865353946292 Email sunkx@ytu.edu.cn; wangaiping@luye.cn hY ${ }_{\mathrm{NC}}$ and incorporate the Creative Commons Attribution - Non Commercial (unported, v3.0) License (http://creativecommons.org/licenses/by-nc/3.0/). By accessing the work you
hereby accept the Terms. Non-commercial uses of the work are permitted without any further permission from Dove Medical Press Limited, provided the work is properly attributed. For permission for commercial use of this work, please see paragraphs 4.2 and 5 of our Terms (https://www.dovepress.com/terms.php).
. 
and drug delivery systems, are required to increase drug efficacy and reduce side effects. Rotigotine is a non-ergot derived D3/D2/D1 agonist that has been reported to exert neuroprotective effects and effectively alleviate the motor symptoms of PD. ${ }^{1,5}$

For brain diseases such as PD and Alzheimer's disease, effective delivery of drugs into the brain is critical for efficacy. However, the blood-brain barrier (BBB), which only allows small lipophilic molecules $(<500 \mathrm{Da})$ to cross, restricts delivery to the brain of a large number of therapeutic agents (apart from certain nutrients, such as hexoses, amino acids, and neuropeptides). ${ }^{6,7}$ Numerous studies have reported attempts to overcome the $\mathrm{BBB} .{ }^{8}$ Intranasal administration has been used to circumvent the BBB and deliver drugs to the brain through the olfactory neuroepithelium via the trigeminal and olfactory nerve pathways noninvasively. ${ }^{9,10}$ Furthermore, nose-to-brain drug delivery has been performed because of its advantages, including reduced drug delivery to nontarget sites, avoidance of hepatic first-pass metabolism, and convenience, for drugs such as rasagiline, alginate, tarenflurbil, and piperine. ${ }^{11-14}$ Therefore, nose-to-brain drug delivery was used in the present study to improve the brainspecific targeting of rotigotine.

Increasing the localization of drugs at the lesion site and reducing levels in other parts of the brain are critical for optimizing drug delivery via intranasal administration. One method used to enhance targeting to the brain is the use of prepared nanoparticles (NPs) modified with biological ligands. Lactoferrin (Lf) is an iron-binding glycoprotein of $80 \mathrm{kDa}$ belonging to the transferring family. ${ }^{15}$ The Lf receptors (LfRs) are highly expressed on the apical surface of respiratory epithelial cells, brain endothelial cells, and neurons. ${ }^{16,17}$ Previous studies demonstrated that neurons accumulate high concentrations of LfRs in PD. ${ }^{16}$ Furthermore, Lf has the ability to cross the $\mathrm{BBB}$, and this property has been widely used for targeted delivery of several drugs to the brain. ${ }^{15,18}$ Therefore, in the current study, we prepared Lf-modified rotigotine NPs (Lf-R-NPs) to investigate whether Lf can improve nose-to-brain delivery of R-NPs after intranasal administration. Lf-R-NPs should be able to rapidly and efficiently deliver rotigotine specifically into the brain. This should help reduce side effects. Liquid extraction surface analysis coupled with tandem mass spectrometry (LESA-MS/MS) is a novel highly sensitive method for assessing the spatial distribution profile of drugs in tissues. LESA-MS/MS shows superior performance and higher sensitivity than other comparable detection methods and can be used to assess the target tissue penetration of drugs.
In our previous study, we prepared Lf-R-NPs (rotigotineloaded mPEG-PLGA NPs surface-modified with Lf) and conducted a preliminary study of their cytotoxicity and cellular uptake. ${ }^{19}$ In the present study, we investigate the distribution of these Lf-R-NPs in different brain tissues following intranasal administration using liquid extraction surface analysis coupled with tandem mass spectrometry LESA-MS/MS. We also examine the pharmacodynamic and neuroprotective properties of these Lf-R-NPs in the rat 6-hydroxydopamine (6-OHDA) model of PD.

\section{Materials and methods \\ Animals}

Sprague Dawley rats (males; 200 \pm 20 g; 4- to 5-week old) were accommodated to a specific pathogen-free environment and supplied with standard rodent chow and water. The rats were housed in steel cages in a room at $22^{\circ} \mathrm{C} \pm 1^{\circ} \mathrm{C}$ under a standard 12-h light/dark cycle. All animal studies were performed according to the Guide for the Care and Use of Laboratory Animals. The Animal Ethics Committee of Yantai University approved the study.

\section{Instruments and reagents}

Rotigotine was kindly provided by Luye Pharma Group (Shandong, People's Republic of China). Bovine Lf, 2-iminothiolane (Traut's reagent), 6-OHDA, apomorphine, and ascorbic acid were purchased from Sigma-Aldrich (St Louis, MO, USA). PEG-PLGA-maleimide (mal-PEGPLGA; 3,400-20,000 Da; 50:50 LA:GA, w/w) and methoxyPEG-PLGA (mPEG-PLGA; 2,000-20,000 Da; 50:50 LA:GA, $\mathrm{w} / \mathrm{w}$ ) were purchased from Polyscitech (West Lafayette, IN, USA). Sephadex G25 and Sepharose CL-4B were purchased from GE Healthcare Bio-Sciences (Piscataway, NJ, USA). All other reagents were of laboratory grade.

\section{Preparation and characterization of R-NPs and Lf-R-NPs}

R-NPs were prepared using the nanoprecipitation method. ${ }^{20}$ Briefly, mal-PEG-PLGA, mPEG-PLGA and rotigotine were dissolved in the organic phase (acetone) and then added dropwise into the aqueous phase (magnetically-stirred under ice-cold conditions) to completely volatilize the organic solvent, followed by filtration through a $0.45-\mu \mathrm{m}$ filter (Millipore, Burlington, MA, USA).

Using Huwyler's method, Lf was thiolated by reaction with a 20-fold molar excess of Traut's reagent for 60 minutes. ${ }^{21}$ The products were purified on a Sephadex G25 column. 
To obtain the Lf-NPs, the purified thiolated Lf was mixed with maleimide-functionalized R-NPs and incubated at room temperature for 9 hours. The product was eluted with $0.01 \mathrm{M}$ phosphate-buffered saline (PBS; pH 7.4) using a $1.5 \times 20 \mathrm{~cm}$ Sepharose CL-4B column to remove the unconjugated protein.

The NPs were characterized for particle size, zeta potential, polydispersity index (PDI), encapsulation efficiency (EE), and loading capacity (LC). The particle size, zeta potential, and PDI were determined by dynamic light scattering method using a Zetasizer NanoZS (Delsa Nano C; Beckman Coulter, Brea, CA, USA). The EE was determined by separating the free drug from the NPs. The concentrations of total drug and free drug were analyzed by high-performance liquid chromatography (LC-20A VP system; Shimadzu, Kyoto, Japan) at $223 \mathrm{~nm}$. The EE and LC of the NPs were calculated using the following equation:

$$
\begin{aligned}
& \mathrm{EE}(\%)=\frac{\text { Total drug }- \text { Free drug }}{\text { Total drug }} \times 100 \\
& \mathrm{LC}(\%)=\frac{\text { Total drug }- \text { Free drug }}{\text { Weight of NPs }} \times 100
\end{aligned}
$$

The measurements were done in triplicate, and the mean \pm SD was calculated.

\section{LESA-MS/MS-based rotigotine biodistribution}

Sprague Dawley rats (males; 200 \pm 20 g; 4- to 5-week old) were randomly divided into two groups for this study. Tissues were harvested for analysis $0.5,1.5,2.5,4$, and 6 hours after intranasal administration of R-NPs and Lf-R-NPs ( $1 \mathrm{mg} / \mathrm{kg}$ rotigotine). The tissues were mounted onto the base plate by polymer adhesive and placed onto the frozen stage of microtome to freeze. Tissue sections ( $40 \mu \mathrm{m}$ thick) were then prepared using a cryomacrotome. The frozen sections were adhered to $1.2-\mathrm{mm}$ thick glass slides $(1 \times 3$ in.) and stored at room temperature ready for LESA. Three rats per group were used for analysis for each time point.

The glass slide with the tissue sample was fixed on a universal adapter plate, and the optical image was scanned using an Epson Perfection V370 Photographic scanner. Coregistration of the picture and spot sampling were performed using LESA Points Software (Advion, Ithaca, NY, USA). The tissue samples and the adapter were placed in a LESA-enabled TriVersa NanoMate robotic system (Advion) without further sample processing. The analytes were extracted from the tissue surface using an electrical conductive pipette tip, and then transferred from the robotic system to an emitter etched on a multichannel chip for nanoelectrospray ionization by infusion. Detection was performed with an MRM 5500 QTRAP mass spectrometer (SCIEX, Concord, Ontario, Canada). The extraction solvent used in all experiments was methanol/water $(8: 2, \mathrm{v} / \mathrm{v})$. At the beginning of each experiment, the pipette tip was automatically calibrated for alignment. The LESA parameters were as follows: the total solvent volume was $1.5 \mu \mathrm{L}$, and $1.1 \mu \mathrm{L}$ was set at a height of $1.8 \mathrm{~mm}$ to form a liquid connector; suction volume was $1.3 \mu \mathrm{L}$, with a suction height of $1.6 \mathrm{~mm}$. The analysis was repeated three times.

\section{Pharmacodynamic study}

Briefly, to prepare the rat model of $\mathrm{PD}$, the animals were given desipramine hydrochloride ( $25 \mathrm{mg} / \mathrm{kg}$, i.p.) 30 minutes before administration of 6-OHDA. To unilaterally lesion the nigrostriatal system, 6-OHDA was injected into the left substantia nigra. After 3 weeks, contralateral rotations were quantified to assess successful establishment of the PD model, as previously reported. ${ }^{22}$ Following the administration of apomorphine hydrochloride $(2 \mathrm{mg} / \mathrm{kg})$, contralateral rotations were recorded in a cylinder with a diameter of $40 \mathrm{~cm}$. Rats exhibiting contralateral rotations of more than $150 \mathrm{r} / 30$ minutes were considered a successful PD model.

The PD rats were randomly divided into four different groups with five animals in each group. Rats administered normal saline via oral administration were used as the negative control. Rats in the L-DOPA group were given Madopar tablets at a dose of $20 \mathrm{mg} / \mathrm{d}$. This group was used as the positive control as this formulation is commercially available for the treatment of PD in humans. Rats in the R-NPs and Lf-R-NPs groups were respectively administered R-NPs and Lf-R-NPs by intranasal administration at a rotigotine dose of $2 \mathrm{mg} / \mathrm{kg} / \mathrm{d}$ (twice a day) without anesthesia or sedation. Each rat was administered in a volume of about $200 \mu \mathrm{L}$. The doses were dispensed using a micropipette $(200 \mu \mathrm{L})$ attached to polyethylene tubing (inner diameter of $0.1 \mathrm{~mm}$ ) at a delivery site positioned $5 \mathrm{~mm}$ deep in each nostril. The rats were placed in a reclined supine position during administration and maintained in this position for about 2 minutes after the dose was administered. The rats were allowed to reacclimate for 30 minutes, placed in a round-bottom pot with a diameter of $40 \mathrm{~cm}$, and contralateral rotations were recorded for 15 minutes. This experiment was performed once a day, for 1 week. 


\section{Immunohistochemistry}

To evaluate the neuroprotective effects of L-DOPA, R-NPs, and Lf-R-NPs, immunohistochemistry for tyrosine hydroxylase (TH) and dopamine transporter (DAT) was performed. Briefly, after the pharmacodynamics experiment, the rats were administered drugs for another 2 weeks and then anesthetized with $10 \%$ trichloroacetaldehyde hydrate $(400 \mathrm{mg} / \mathrm{kg}$, intraperitoneally). The rats were perfused with $200 \mathrm{~mL}$ PBS

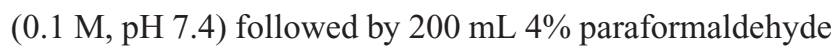
until the limbs were stiff. The brains were removed, fixed with $4 \%$ paraformaldehyde for 24 hours, dehydrated, and embedded in paraffin and sliced into $4-\mu \mathrm{m}$ sections. After dewaxing and rehydration, the sections were incubated with $0.3 \% \mathrm{H}_{2} \mathrm{O}_{2}$ and blocked with 5\% normal goat serum, followed by incubation with rabbit anti-TH monoclonal antibody (1:300) or rabbit anti-DAT monoclonal antibody (1:300) for 1 hour at room temperature. Subsequently, the sections were incubated for 10 minutes with goat anti-mouse IgG secondary antibody (1:300). Finally, the sections were stained using a DAB kit, placed under a coverslip, and images were captured under a microscope. The images were analyzed using Image-Pro Plus 6.0 software (Media Cybernetics, Rockville, MD, USA).

\section{Statistical analysis}

All values are presented as mean \pm SD. One-way analysis of variance was used to compare differences between groups using SPSS 22.0 software (IBM Corp., Armonk, NY, USA). $P<0.05$ and $P<0.01$ were considered to be statistically significant and highly significant, respectively.

\section{Results and discussion}

\section{Preparation and characterization of} R-NPs and Lf-R-NPs

We prepared NPs using the formulation optimized in our group's previous studies. ${ }^{19}$ The NPs were characterized for particle size, zeta potential, PDI, EE, and LC (Table 1). The mean particle size of the NPs was not more than $200 \mathrm{~nm}$, making them suitable for nose-to-brain delivery. ${ }^{23}$ The zeta potential was about $-20 \mathrm{mV}$, indicating that the NPs were

Table I Characterization of R-NPs and Lf-R-NPs

\begin{tabular}{lllll}
\hline Formulation & $\begin{array}{l}\text { Particle } \\
\text { size, } \mathbf{n m}\end{array}$ & $\begin{array}{l}\text { Zeta potential, } \\
\mathbf{m V}\end{array}$ & EE, \% & LC, \% \\
\hline R-NPs & $62.4 \pm 3.9$ & $-22.48 \pm 1.27$ & $91.23 \pm 4.28$ & $8.29 \pm 0.04$ \\
Lf-R-NPs & $118.4 \pm 12.4$ & $-21.94 \pm 2.83$ & $88.07 \pm 6.01$ & $8.01 \pm 0.05$ \\
\hline
\end{tabular}

Abbreviations: EE, encapsulation efficiency; LC, loading capacity; Lf-R-NPs, lactoferrin-modified rotigotine nanoparticles; R-NPs, rotigotine nanoparticles. stable. The EE and LC were about $90 \%$ and $8 \%$, respectively. These results are consistent with our previous reports.

\section{Rotigotine biodistribution assessed by LESA-MS/MS}

The tissue sections were analyzed directly by LESA-MS/MS after intranasal administration of R-NPs and Lf-R-NPs $(1 \mathrm{mg} / \mathrm{kg}$ rotigotine), and the relative concentrations of rotigotine were estimated and averaged (assay performed in triplicate; MRM assay mode) (Figure 1). The LESA-MS/MS analysis revealed that rotigotine had accumulated mainly in brain tissues, indicating that R-NPs and Lf-R-NPs are targeted preferentially to the brain after intranasal administration. The signal at 0.5 hour shows that R-NPs and Lf-R-NPs can rapidly be transported to the brain. However, the highest signal for Lf-R-NPs was at 1.5 hours in brain tissues, indicating that Lf-R-NPs delivered a higher sustained amount of the drug compared with R-NPs. Lf-R-NPs are likely transported to the brain across the nasal mucosa both by passive transport, similar to R-NPs, as well as by Lf receptor-mediated transport, resulting in relatively high sustained levels of drug in the brain. Furthermore, Lf-RNPs were better able to target the striatum than R-NPs, suggesting that Lf helps target dopamine neurons (Figure 1C). In liver and kidney tissues, the rotigotine signals were low at every time point examined, for both R-NPs and Lf-R-NPs.

LESA-MS/MS was used to further investigate the spatial biodistribution of rotigotine in the brain in rats administered Lf-R-NPs. The signal intensity in olfactory bulb tissue sections was strong, indicating that rotigotine was directly delivered to the brain via the olfactory and trigeminal nerve pathways following intranasal administration (Figure 2). Furthermore, the signal intensity was stronger in the striatum than in the hippocampus. These results further demonstrate that Lf-R-NPs are able to selectively target the striatum.

\section{Pharmacodynamic study}

The therapeutic efficacy of the NPs for PD after intranasal administration was evaluated by measuring contralateral rotations. The body weights after surgery were not significantly different between the groups. There was no significant difference in rotational behavior before surgery between the groups. However, PD rats exhibited significantly greater apomorphine-induced contralateral rotations after surgery compared with the normal group ( $P<0.01$; Figure 3$)$. Interestingly, there was no significant difference in contralateral rotations after administration between the L-DOPA and 


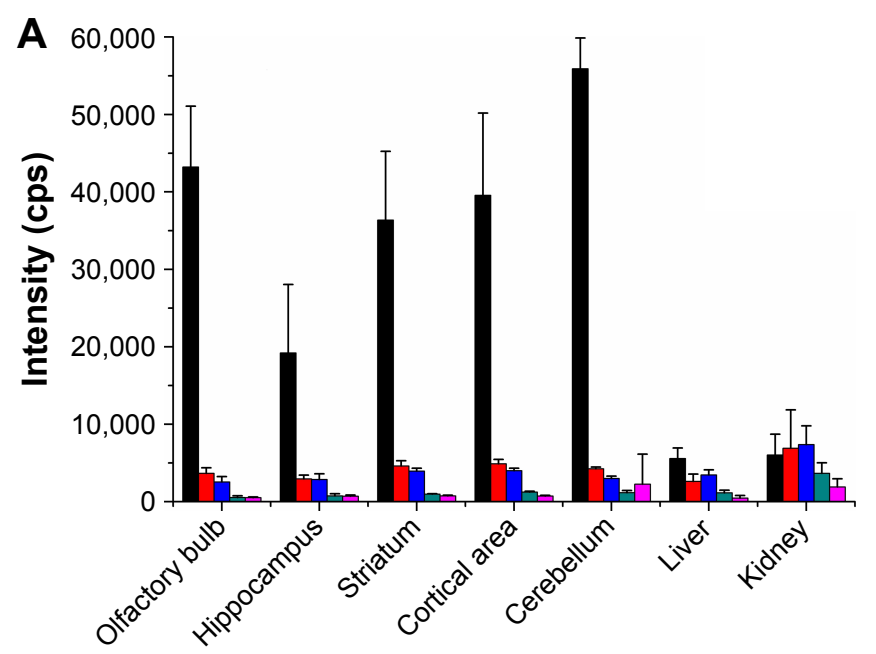

Tissue

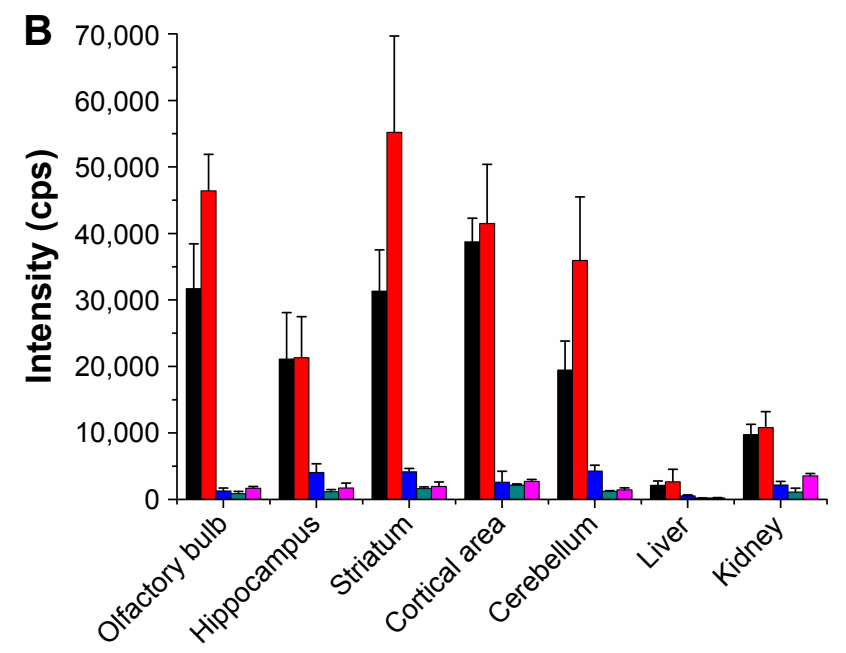

Tissue

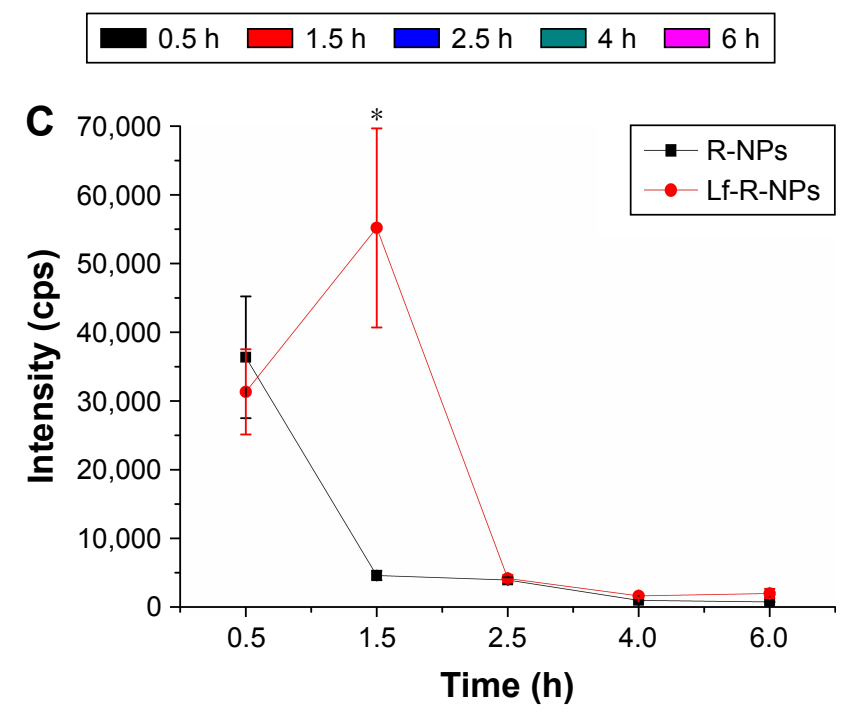

Figure I The rotigotine biodistribution in tissues determined by LESA-MS/MS.

Notes: (A) Mean rotigotine signal $(n=3)$ in tissues after intranasal R-NPs administration. (B) Mean rotigotine signal $(n=3)$ in tissues after intranasal Lf-R-NPs administration. (C) Mean rotigotine signal $(n=3)$ in striatum after intranasal R-NPs and Lf-R-NPs administration. $* P<0.05$ compared with R-NPs.

Abbreviations: LESA-MS/MS, liquid extraction surface analysis coupled with tandem mass spectrometry; Lf-R-NPs, lactoferrin-modified rotigotine nanoparticles; R-NPs, rotigotine nanoparticles.

R-NPs groups in the first 3 days, while a significant difference was observed between the L-DOPA and Lf-R-NPs groups on the second and third days $(P<0.05)$. After 1 week of administration, there was a notable change in contralateral rotations. Statistical analysis of the contralateral rotations over a 15 -min period revealed a significant difference between the L-DOPA and R-NPs groups $(P<0.05)$. In addition, a significant difference was found between the Lf-R-NPs and R-NPs groups $(P<0.05)$. These results indicate that both R-NPs and Lf-R-NPs can deliver effective doses of rotigotine into the brain. Furthermore, the efficiency of Lf-R-NPs was greater than that of R-NPs, suggesting that a greater amount of drug was delivered to the target, likely achieved by the Lf modification on the surface of the R-NPs.

\section{Immunohistochemistry for TH and DAT}

We also examined the expression of TH and DAT to assess the neuroprotective effects of R-NPs and Lf-R-NPs. The neuroprotective effect on the substantia nigra and striatum was determined by calculating the ratio of the average relative optical density (A-value) of the lesioned side (L side) and the unlesioned side ( $\mathrm{R}$ side) using Image-Pro Plus 6.0 software (Media Cybernetics).

The TH immunohistochemistry results are shown in Figures 4 and 5. Both R-NPs and Lf-R-NPs significantly 


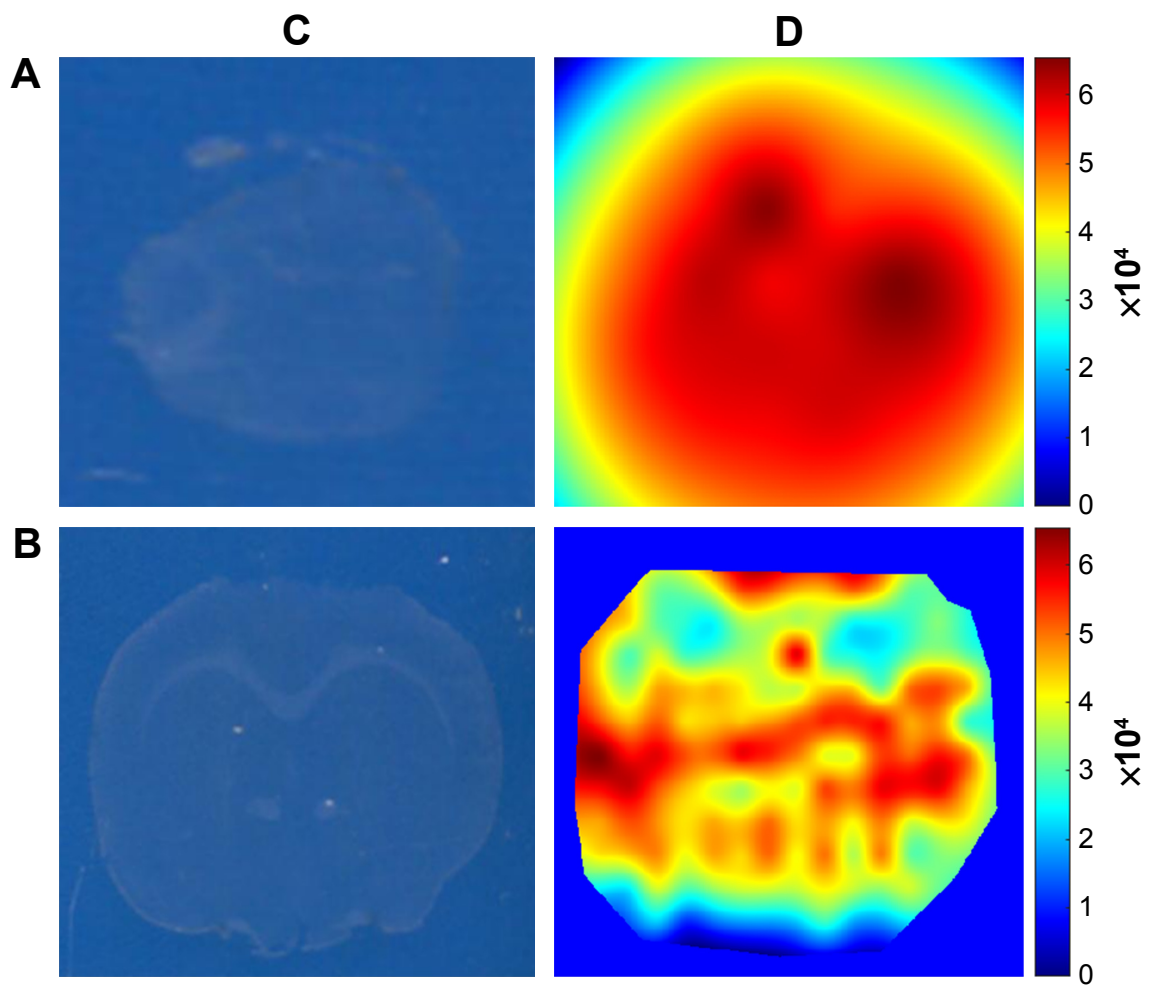

Figure 2 The spatial biodistribution of rotigotine in the brain in rats administered Lf-R-NPs.

Notes: (A) LESA-MS/MS images of olfactory bulbs tissue sections after Lf-R-NPs dosing 0.5 hour. (B) LESA-MS/MS images of brain tissue sections after Lf-R-NPs dosing 0.5 hour. (C) Optical image obtained using an Epson Perfection V370 Photo scanner. (D) The calculated ion intensity heat map from Matlab.

Abbreviations: LESA-MS/MS, liquid extraction surface analysis coupled with tandem mass spectrometry; Lf-R-NPs, lactoferrin-modified rotigotine nanoparticles; R-NPs, rotigotine nanoparticles.

upregulated $\mathrm{TH}$ expression and reduced dopaminergic neuronal loss in the substantia nigra following 6-OHDA injection $(P<0.05$, compared with the L-DOPA group). Moreover, Lf-R-NPs had a significantly greater neuroprotective effect

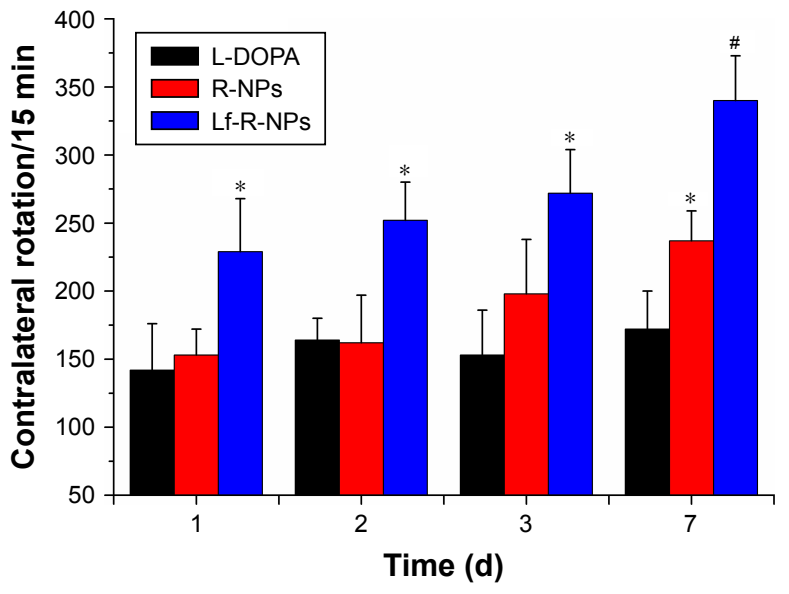

Figure 3 Contralateral rotations of PD rats every 15 minutes in the L-DOPA, R-NPs, and Lf-R-NPs groups.

Notes: ${ }^{* P}<0.05$ compared with L-DOPA group. ${ }^{\# P}<0.05$ compared with R-NPs group.

Abbreviations: L-DOPA, levodopa; Lf-R-NPs, lactoferrin-modified rotigotine nanoparticles; PD, Parkinson's disease; R-NPs, rotigotine nanoparticles. than R-NPs $(P<0.05)$. There was a significant difference in A-values in the striatum between the Lf-R-NPs and R-NPs groups $(P<0.01$; Figure 5).

We also found a substantial decrease in DAT immunoreactivity in the 6-OHDA-injected rats. Treatment with R-NPs and Lf-R-NPs significantly restored DAT immunoreactivity in the substantia nigra compared with the L-DOPA group (Figure 6). Interestingly, there was no significant difference in DAT immunoreactivity between the L-DOPA and R-NPs groups. However, there was a very significant difference in DAT immunoreactivity between the R-NPs and Lf-R-NPs groups $(P<0.01)$. And also, there was a significant difference in A-values in the striatum between the Lf-R-NPs and R-NPs groups $(P<0.01$; Figure 7). These results suggest that rotigotine has neuroprotective effects and further demonstrate that Lf-modified R-NPs can deliver more rotigotine into the brain to more effectively protect nigrostriatal dopaminergic neurons.

\section{Conclusion}

Lf-R-NPs were successfully prepared in this study, and LESA-MS/MS was successfully used to assess their 

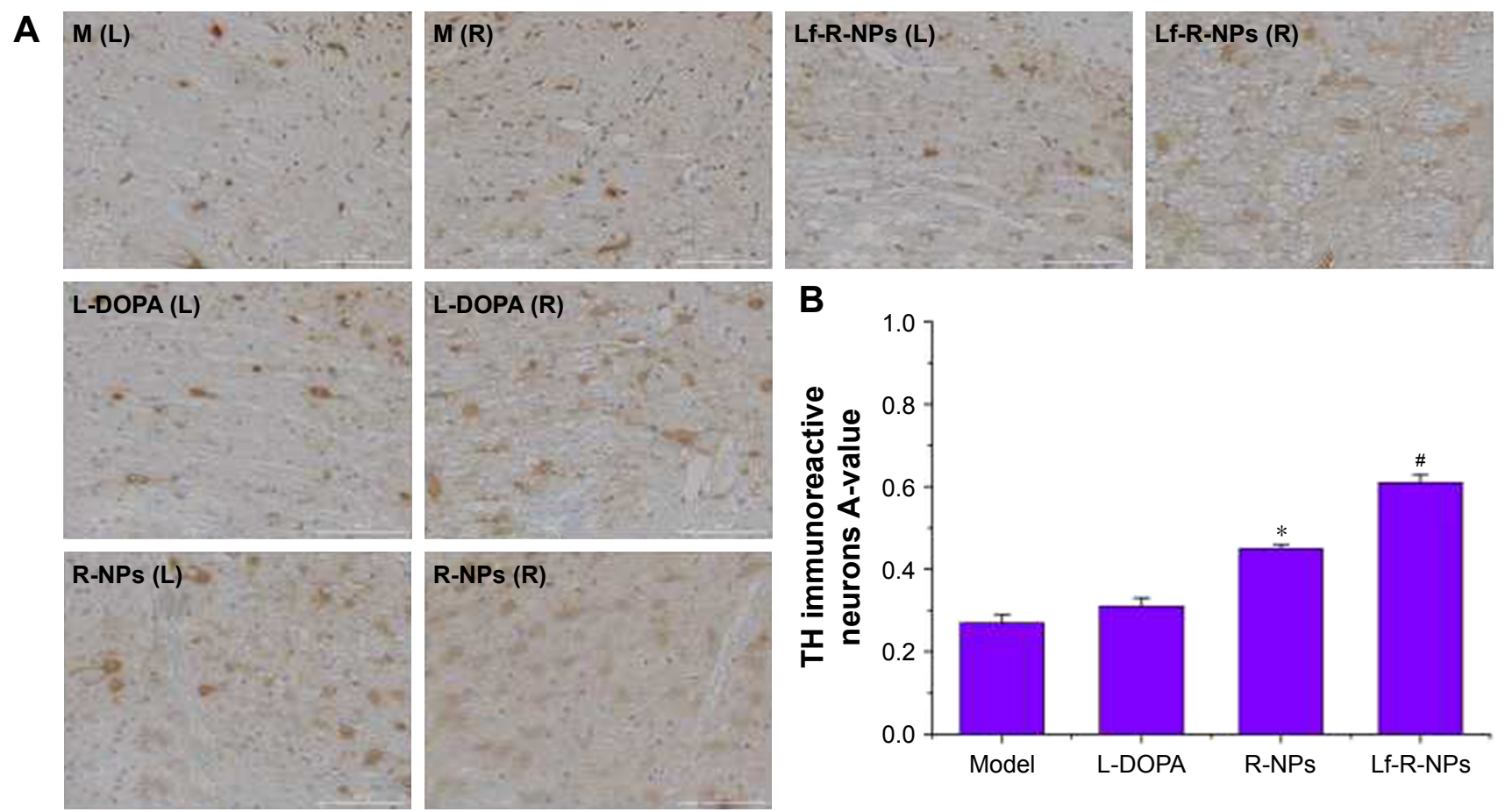

Figure 4 TH expression by immunostaining in the substantia nigra.

Notes: (A) Representative images of TH immunostainng in the substantia nigra; (B) Data were analyzed by counting the relative optical density value (A-value) using Image-Pro

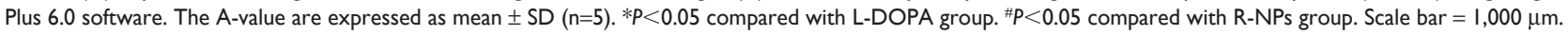
Abbreviations: L-DOPA, levodopa; R-NPs, rotigotine nanoparticles; TH, tyrosine hydroxylase; M, model; L, left; R, right.
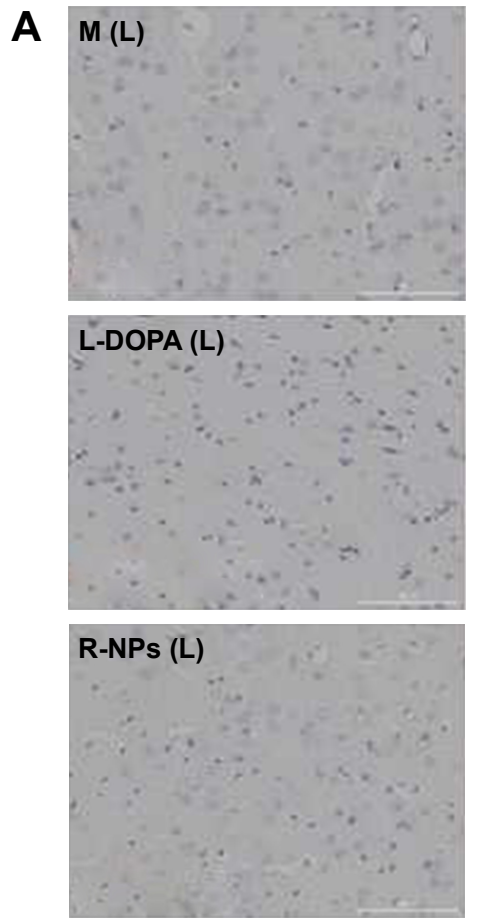
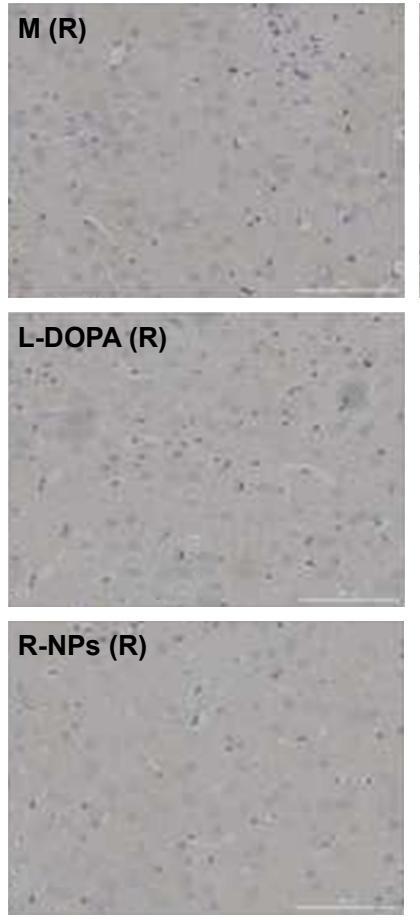
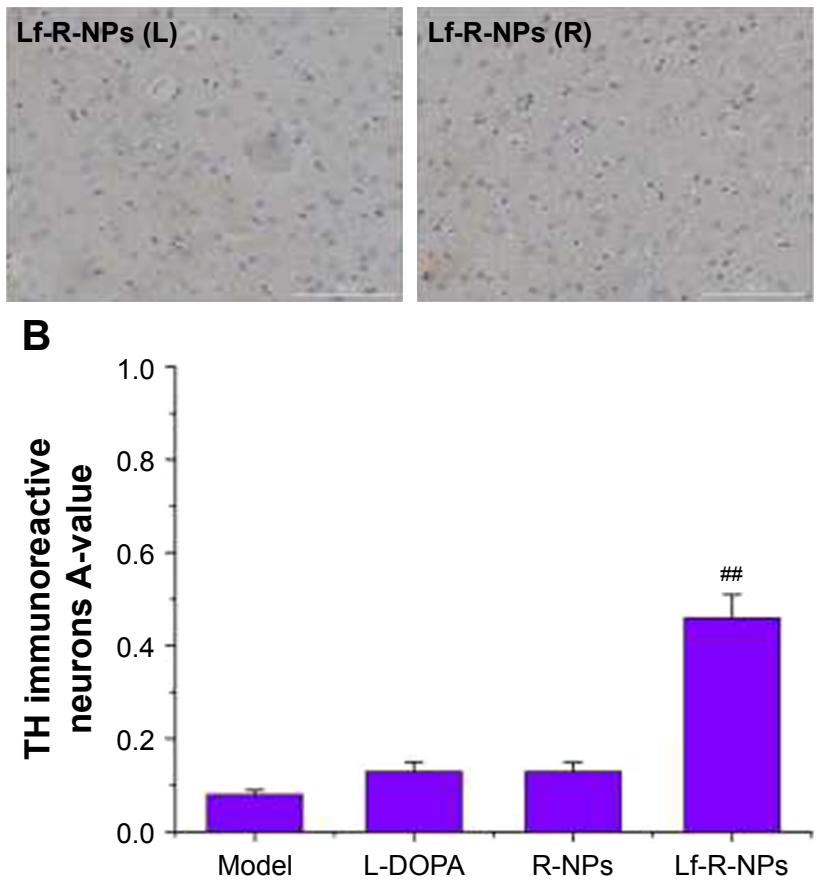

Figure $\mathbf{5}$ TH expression by immunostaining in striatum.

Notes: (A) Representative images of TH immunostainng in the striatum of rats; (B) Data were analyzed by counting the relative optical density value (A-value) using Image-Pro Plus 6.0 software. The A-value are expressed as mean \pm SD $(n=5) .{ }^{\prime} P<0.01$ compared with $R-N P s$ group. Scale bar $=1,000 \mu$ m.

Abbreviations: L-DOPA, levodopa; R-NPs, rotigotine nanoparticles; TH, tyrosine hydroxylase; M, model; L, left; R, right. 

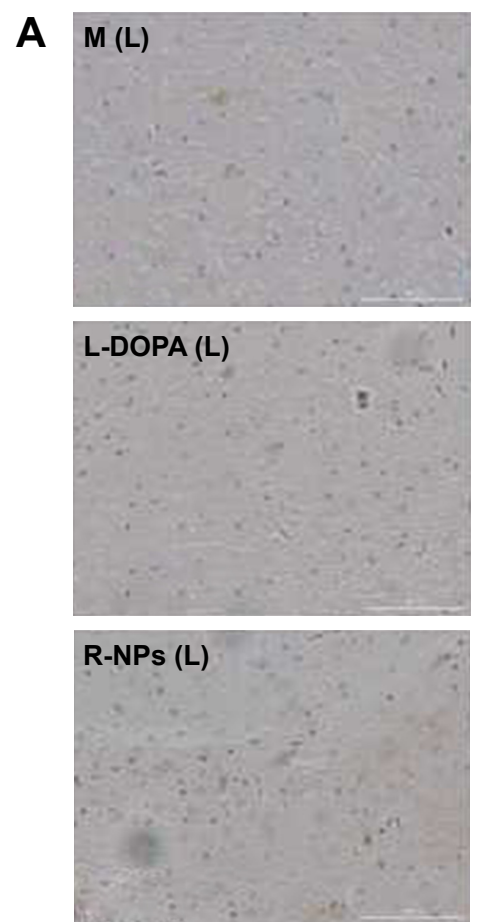
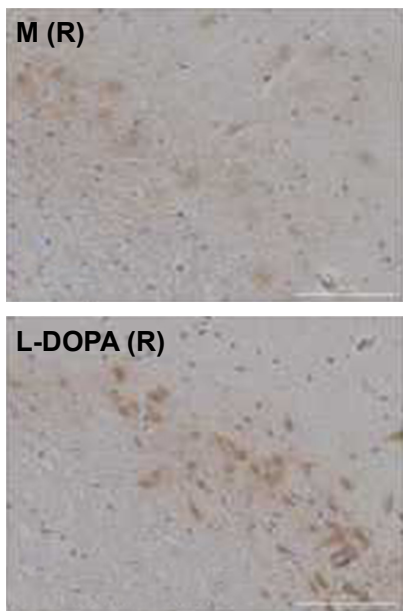

R-NPs (R)
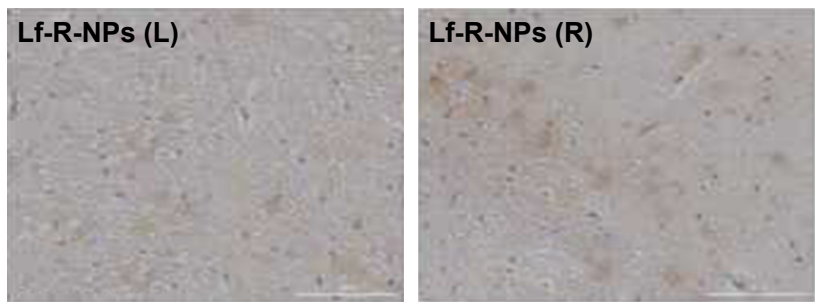

B

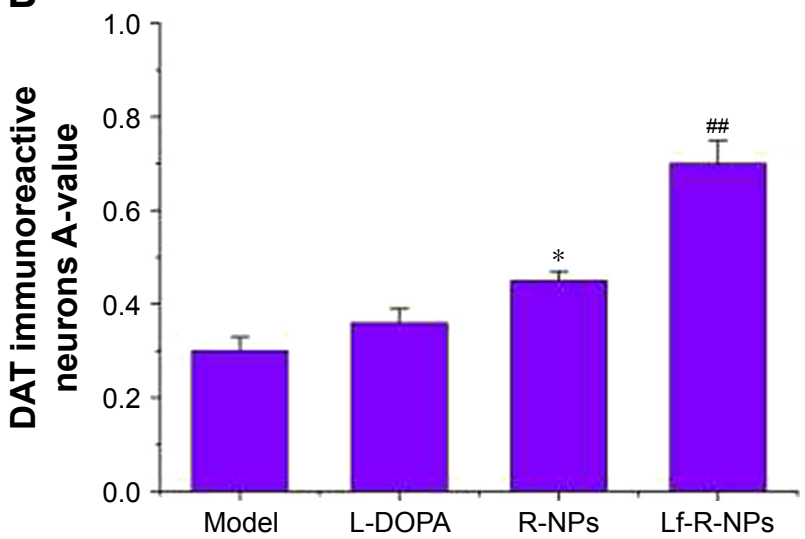

Figure 6 DAT expression by immunostaining in substantia nigra.

Notes: (A) Representative images of DAT immunostainng in the substantia nigra of rats; (B) Data were analyzed by counting the relative optical density value (A-value) using Image-Pro Plus 6.0 software. The A-value are expressed as mean \pm SD ( $n=5)$. $* P<0.05$ compared with L-DOPA group. ${ }^{\# P<0.01}$ compared with R-NPs group. Scale bar $=1,000 \mu \mathrm{m}$

Abbreviations: DAT, dopamine transporter; L-DOPA, levodopa; R-NPs, rotigotine nanoparticles; M, model; L, left; R, right.
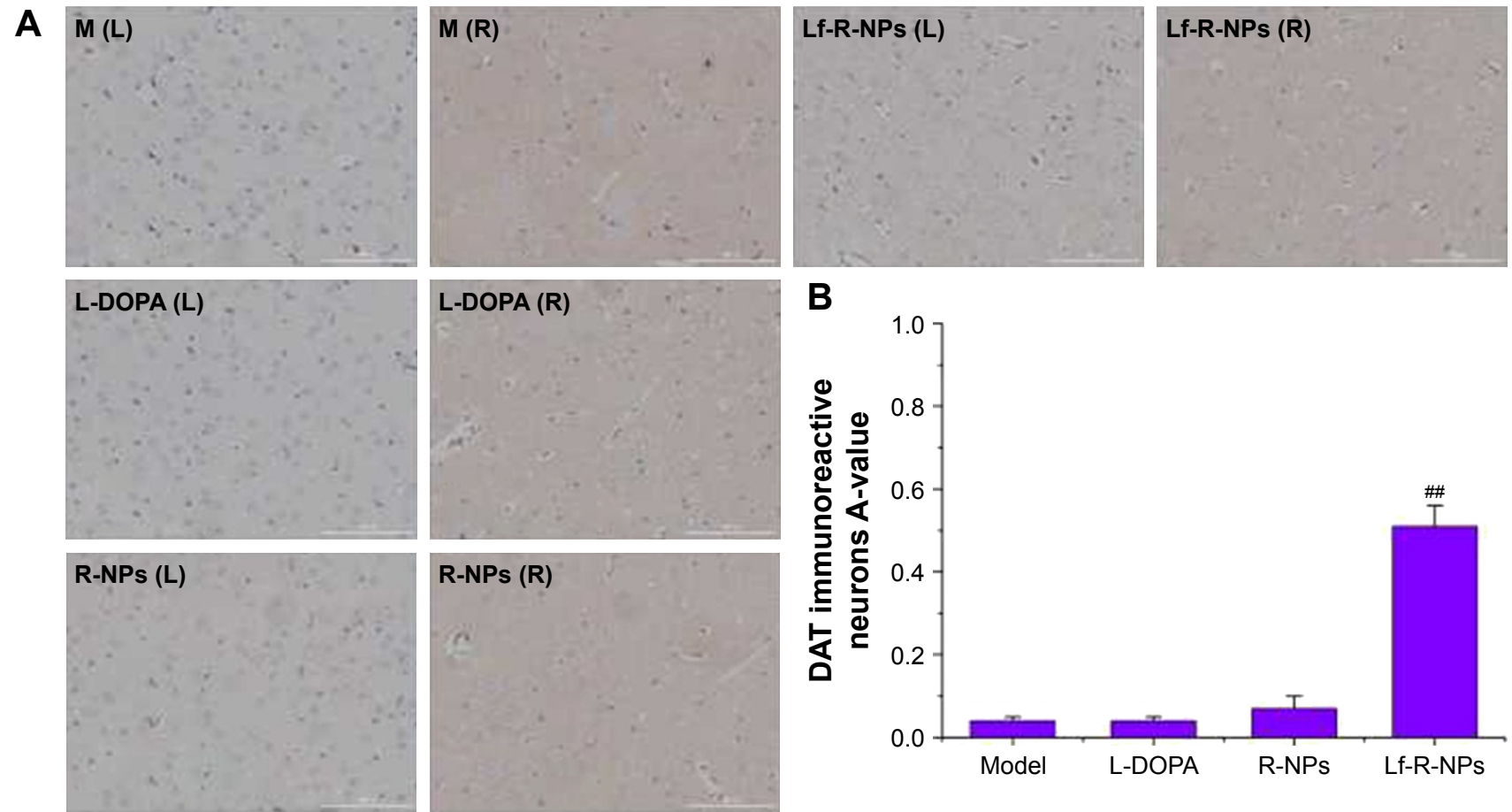

Figure 7 DAT expression by immunostaining in the striatum.

Notes: (A) Representative images of DAT immunostainng in the striatum of rats; (B) Data were analyzed by counting the relative optical density value (A-value) using Image-Pro Plus 6.0 software. The A-value are expressed as mean \pm SD $(n=5)$. ${ }^{\#} P<0.0$ I compared with R-NPs group. Scale bar $=I, 000 \mu m$.

Abbreviations: DAT, dopamine transporter; L-DOPA, levodopa; R-NPs, rotigotine nanoparticles; M, model; L, left; R, right. 
biodistribution. Lf-R-NPs rapidly entered the brain and displayed a better sustained-release effect compared with R-NPs. In addition, Lf-R-NPs showed comparatively better efficacy in delivering rotigotine to the brain in the rat 6-OHDA model of PD rats, based on the pharmacodynamic study. Furthermore, Lf-R-NPs significantly alleviated nigrostriatal dopaminergic neurodegeneration. Further studies are required to more fully evaluate the efficacy and risks of this drug preparation for clinical application.

\section{Acknowledgments}

This study was funded by the Natural Science Foundation of Shandong Province (ZR2014HM062) and Science and Technology Project of Higher Education of Shandong Province (J16LM52).

\section{Disclosure}

The authors report no conflicts of interest in this work.

\section{References}

1. Yu X, Yao JY, He J, Tian JW. Protection of MPTP-induced neuroinflammation and neurodegeneration by rotigotine-loaded microspheres. Life Sci. 2015;124:136-143.

2. Hao XM, Li LD, Duan CL, Li YJ. Neuroprotective effect of alphamangostin on mitochondrial dysfunction and alpha-synuclein aggregation in rotenone-induced model of Parkinson's disease in differentiated SH-SY5Y cells. J Asian Nat Prod Res. 2017;19(8):833-845.

3. Siracusa R, Paterniti I, Cordaro M, et al. Neuroprotective effects of Temsirolimus in animal models of Parkinson's disease. Mol Neurobiol. Epub 2017 March 29.

4. McAfee DA, Hadgraft J, Lane ME. Rotigotine: the first new chemical entity for transdermal drug delivery. Eur J Pharmaceut Biopharmaceut. 2014;88(3):586-593.

5. Stockwell KA, Scheller DK, Smith LA, et al. Continuous rotigotine administration reduces dyskinesia resulting from pulsatile treatment with rotigotine or L-DOPA in MPTP-treated common marmosets. Exp Neurol. 2010;221(1):79-85.

6. Gao H, Pang Z, Jiang X. Targeted delivery of nano-therapeutics for major disorders of the central nervous system. Pharmaceut Res. 2013; 30(10):2485-2498.

7. Zhang L, Han L, Qin J, Lu W, Wang J. The use of borneol as an enhancer for targeting aprotinin-conjugated PEG-PLGA nanoparticles to the brain. Pharmaceut Res. 2013;30(10):2560-2572.
8. Zong T, Mei L, Gao H, et al. Enhanced glioma targeting and penetration by dual-targeting liposome co-modified with T7 and TAT. JPharm Sci. 2014;103(12):3891-3901.

9. Ali J, Ali M, Baboota S, et al. Potential of nanoparticulate drug delivery systems by intranasal administration. Curr Pharm Des. 2010;16: $1644-1653$.

10. Lalani J, Baradia D, Lalani R, Misra A. Brain targeted intranasal delivery of tramadol: comparative study of microemulsion and nanoemulsion. Pharm Dev Technol. 2014:1-10.

11. Mittal D, Md S, Hasan Q, et al. Brain targeted nanoparticulate drug delivery system of rasagiline via intranasal route. Drug Deliv. 2016; 23(1):130-139.

12. Haque S, Md S, Sahni JK, Ali J, Baboota S. Development and evaluation of brain targeted intranasal alginate nanoparticles for treatment of depression. J Psychiatr Res. 2014;48(1):1-12.

13. Muntimadugu E, Dhommati R, Jain A, Challa VG, Shaheen M, Khan W. Intranasal delivery of nanoparticle encapsulated tarenflurbil: a potential brain targeting strategy for Alzheimer's disease. Eur J Pharm Sci. 2016;92:224-234.

14. Elnaggar YS, Etman SM, Abdelmonsif DA, Abdallah OY. Intranasal piperine-loaded Chitosan nanoparticles as brain-targeted therapy in Alzheimer's disease: optimization, biological efficacy, and potential toxicity. J Pharm Sci. 2015;104(10):3544-3556.

15. Kumari S, Ahsan SM, Kumar JM, Kondapi AK, Rao NM. Overcoming blood brain barrier with a dual purpose Temozolomide loaded Lactoferrin nanoparticles for combating glioma (SERP-17-12433). Sci Rep. 2017;7(1):6602.

16. Elfinger M, Maucksch C, Rudolph C. Characterization of lactoferrin as a targeting ligand for nonviral gene delivery to airway epithelial cells. Biomaterials. 2007;28(23):3448-3455.

17. Suzuki YA, Lopez V, Lonnerdal B. Mammalian lactoferrin receptors: structure and function. Cell Mol Life Sci. 2005;62(22):2560-2575.

18. Kumar P, Lakshmi YS, C B, Golla K, Kondapi AK. Improved safety, bioavailability and pharmacokinetics of zidovudine through lactoferrin nanoparticles during oral administration in rats. PLoS One. 2015;10(10): e0140399.

19. Bi C, Wang A, Chu Y, et al. Intranasal delivery of rotigotine to the brain with lactoferrin-modified PEG-PLGA nanoparticles for Parkinson's disease treatment. Int J Nanomed. 2016;11:6547-6559.

20. Sharma D, Sharma RK, Sharma N, et al. Nose-to-brain delivery of PLGAdiazepam nanoparticles. AAPS PharmSciTech. 2015;16(5):1108-1121.

21. Liu Z, Jiang M, Kang T, et al. Lactoferrin-modified PEG-co-PCL nanoparticles for enhanced brain delivery of NAP peptide following intranasal administration. Biomaterials. 2013;34(15):3870-3881.

22. Afshin-Majd S, Khalili M, Roghani M, Mehranmehr N, Baluchnejadmojarad T. Carnosine exerts neuroprotective effect against 6-hydroxydopamine toxicity in hemiparkinsonian rat. Mol Neurobiol. 2015;51(3):1064-1070.

23. Mistry A, Stolnik S, Illum L. Nanoparticles for direct nose-to-brain delivery of drugs. Int J Pharm. 2009;379(1):146-157.
International Journal of Nanomedicine

\section{Publish your work in this journal}

The International Journal of Nanomedicine is an international, peerreviewed journal focusing on the application of nanotechnology in diagnostics, therapeutics, and drug delivery systems throughou the biomedical field. This journal is indexed on PubMed Central, MedLine, CAS, SciSearch ${ }^{\circledR}$, Current Contents ${ }^{\circledR} /$ Clinical Medicine,

\section{Dovepress}

Journal Citation Reports/Science Edition, EMBase, Scopus and the Elsevier Bibliographic databases. The manuscript management system is completely online and includes a very quick and fair peer-review system, which is all easy to use. Visit http://www.dovepress.com/ testimonials.php to read real quotes from published authors. 